\title{
Rotation modulation scheme for the restraint of carrier maneuver and gyro scale factor error
}

\author{
Jiaxing $\mathrm{Zhao}^{1}$, Lei Wang $^{1, *}$ and Guohua Su${ }^{1}$
}

${ }^{1}$ School of Instrument Science and opto electronic engineering, Beihang University, Beijing City, Haidian District Xueyuan Road No. 37 China

\section{*17wanglei@buaa.edu.cn}

Keywords: Rotation, Inertial navigation, Coordinate system, Scheme

Abstract. Rotating inertial navigation system(RINS) using rotating modulation technology to compensate the error of inertial sensors and it can improve the precision of the navigation system. Rotation modulation scheme, including rotation coordinate system and rotation direction, plays a key role in RINS. In this paper, a brief analysis of rotation modulation error restrain mechanism is made firstly, then analysis of rotating coordinate and rotation direction are conducted, finally computer simulation is made to verify it. The results show that the rotation scheme around geographic coordinate has obvious advantages than that around the carrier coordinate, and the rotation scheme of forward-reverse-reverse-forward has higher navigation accuracy than the scheme of forward-reverse.

\section{Introduction}

The inertial navigation system has the advantages of working independently, providing all the navigation information and strong anti-interference ability, but it also has the defect that positioning errors accumulate over time ${ }^{[1]}$. In fact, the general scheme is taking system error self compensation technique or improving the precision of inertial sensors, to improve the precision of navigation system $^{2]}$. The latter is more costly, while the former is a cost-effective way to achieve high-precision programs, which has been extensively researched and applied.

Single axis RINS is one of the realization of system error self compensation technique. It utilizes the rotation of the heading axis to automatically compensate the constant error of the inertial sensors in the horizontal direction ${ }^{[3,4]}$. Therefore, it is appropriate for a large number of situations with heading movements and little change in attitude, such as seafaring or vehicles ${ }^{[5]}$. In this paper, to improve the navigation accuracy of the system, I optimized the technical details of single axis RINS through modeling and simulation of single axis rotation error suppression technique.

\section{Principle of Single axis RINS}

I take the gyro error as an example to summarize the principle of single axis RINS. Error propagation equation of strap down inertial navigation is shown as equation ${ }^{[6]}$ :

$$
\dot{\phi}=-\omega_{i n}^{n} \times \phi+\delta \omega_{i n}^{n}-C_{b}^{n} \delta \omega_{i b}^{b}
$$

Because the output value of gyro in RINS is measured in a rotating coordinate system, there is one more rotation we should take in the navigation solution. The error propagation equation become:

$$
\dot{\phi}=-\omega_{i n}^{n} \times \phi+\delta \omega_{i n}^{n}-C_{b}^{n} C_{p}^{b} \delta \omega_{i p}^{p}
$$

As IMU rotates along z-axis in body axis system, the transition matrix from system(p) to (b) is: 


$$
C_{p}^{b}(t)=\left[\begin{array}{ccc}
\cos \omega t & -\sin \omega t & 0 \\
\sin \omega t & \cos \omega t & 0 \\
0 & 0 & 1
\end{array}\right]
$$

$\delta \omega_{i p}^{p}=\left[\delta \omega_{i p}^{p x}, \delta \omega_{i p}^{p y}, \delta \omega_{i p}^{p z}\right], \delta f_{i p}^{p}=\left[\delta f_{i p}^{p x}, \delta f_{i p}^{p y}, \delta f_{i p}^{p z}\right]$, Only consider the stationary base situation ( $C_{b}^{n}$ is constant matrix). And the measuring error caused by gyro bias after rotations is:

$$
\delta \omega^{n}=C_{b}^{n} C_{p}^{b} \delta \omega_{i p}^{p}=C_{b}^{n}\left[\begin{array}{ccc}
\cos \omega t & -\sin \omega t & 0 \\
\sin \omega t & \cos \omega t & 0 \\
0 & 0 & 1
\end{array}\right]\left[\begin{array}{l}
\delta \omega_{i p}^{p x} \\
\delta \omega_{i p}^{p y} \\
\delta \omega_{i p}^{p z}
\end{array}\right]=C_{b}^{n}\left[\begin{array}{c}
\delta \omega_{i p}^{p x} \cos \omega t-\delta \omega_{i p}^{p y} \sin \omega t \\
\delta \omega_{i p}^{p x} \sin \omega t+\delta \omega_{i p}^{p y} \cos \omega t \\
\delta \omega_{i p}^{p z}
\end{array}\right]
$$

From the above equation, the error in the horizontal direction of the inertial element is modulated by the sine and cosine. It presents a cyclical change, with a mean value of zero. Therefore, by rotating the IMU single axis rotation, the output error of the inertial sensors can be averaged and suppressed during the navigation solution, and the navigation accuracy of the system can be improved.

\section{Design of rotation coordinate system}

The inhibitory effect of navigation error is different, when RINS rotates along different coordinate systems. If IMU rotates along the body axis system with $\omega_{1}$, the measuring error of gyro is:

$$
\delta \omega^{n}=C_{b}^{n} C_{p}^{b} \delta \omega_{i p}^{p}=C_{b}^{n}\left[\begin{array}{c}
\delta \omega_{i p}^{p x} \cos \omega_{1} t-\delta \omega_{i p}^{p y} \sin \omega_{1} t \\
\delta \omega_{i p}^{p x} \sin \omega_{1} t+\delta \omega_{i p}^{p y} \cos \omega_{1} t \\
\delta \omega_{i p}^{p z}
\end{array}\right]
$$

Rotation can modulate the constant bias which is perpendicular to the rotating shaft, only if the $C_{b}^{n}$ is constant matrix. The course change of single axis RINS is very common, so $C_{b}^{n}$ cannot be a constant matrix. It is an extreme situation that the carrier rotates along the heading axis with $-\omega_{1}$, in which the measuring error is:

$$
\delta \omega^{n}=\left[\begin{array}{ccc}
\cos \omega_{1} t & \sin \omega_{1} t & 0 \\
-\sin \omega_{1} t & \cos \omega_{1} t & 0 \\
0 & 0 & 1
\end{array}\right]\left[\begin{array}{c}
\delta \omega_{i p}^{p x} \cos \omega_{1} t-\delta \omega_{i p}^{p y} \sin \omega_{1} t \\
\delta \omega_{i p}^{p x} \sin \omega_{1} t+\delta \omega_{i p}^{p y} \cos \omega_{1} t \\
\delta \omega_{i p}^{p z}
\end{array}\right]=\left[\begin{array}{l}
\delta \omega_{i p}^{p x} \\
\delta \omega_{i p}^{p y} \\
\delta \omega_{i p}^{p z}
\end{array}\right]
$$

Rotation modulation is completely failure. It is obvious in physical process, because IMU is static to the navigation coordinate systems in this situation. More generally, the carrier rotates along the heading axis with $\omega_{2}$. At this point, the measuring error is:

$$
\delta \omega^{n}=\left[\begin{array}{c}
\cos \left(\omega_{1}+\omega_{2}\right) t \cdot \delta \omega_{i p}^{p x}-\sin \left(\omega_{1}+\omega_{2}\right) t \cdot \delta \omega_{i p}^{p y} \\
\sin \left(\omega_{1}+\omega_{2}\right) t \cdot \delta \omega_{i p}^{p x}+\cos \left(\omega_{1}+\omega_{2}\right) t \cdot \delta \omega_{i p}^{p y} \\
\delta \omega_{i p}^{p z}
\end{array}\right]
$$

From the above equation, we can know that if the carrier rotates along the heading axis with the constant angular velocity $\omega_{2}$, the constant bias of gyro can be modulated in equivalent rotation period $2 \pi /\left(\omega_{1}+\omega_{2}\right)$ in navigation coordinate system. In other word, when $\omega_{1}$ and $\omega_{2}$ are in the same 
direction, equivalent modulation period become shorter, and when they are in the different direction, the period become longer. It just seems that modulation period is changed, which has little effect on the result.

But rotation scheme in practical situation is combined with forward and reverse rotation because of the scale factor error. When the carrier rotates along the heading axis with constant angular velocity, the period of forward rotation is different from that of forward rotation, which cannot eliminate the influence of constant drift on navigation accuracy.

When the carrier is rotated along the heading axis with the changing angular velocity, the constant error of the gyro cannot be modulated into a positive. For example, heading rotation just appear in the positive half cycle of motor rotation. In a positive and negative rotation modulation cycle, the error of the normal value of the gyro accelerometer cannot cancel each other.

If IMU rotates along the geographic coordinate system with $\omega_{1}$, the measuring error of gyro is:

$$
\delta \omega^{n}=C_{p}^{n} \delta \omega_{i p}^{p}=\left[\begin{array}{c}
\delta \omega_{i p}^{p x} \cos \omega t-\delta \omega_{i p}^{p y} \sin \omega t \\
\delta \omega_{i p}^{p x} \sin \omega t+\delta \omega_{i p}^{p y} \cos \omega t \\
\delta \omega_{i p}^{p z}
\end{array}\right]
$$

The error suppression effect is not affected by the rotation of the carrier, which can completely eliminate the constant bias of gyro perpendicular to the rotating shaft.

In the example of a single axis forward and reverse rotation, the computer simulation is carried out to compare the influence on the navigation accuracy between rotating along the geographic coordinate and the carrier coordinate system.

Simulation condition: Gyro bias $0.01 \%$. IMU $\omega_{1}=6 \%$. Carrier heading changing is reverse uniform rotation $180^{\circ} . \omega_{2}=4^{\circ} / \mathrm{s}$. Simulation time 30min. Heading rotation appears in the middle of the simulation(10min).
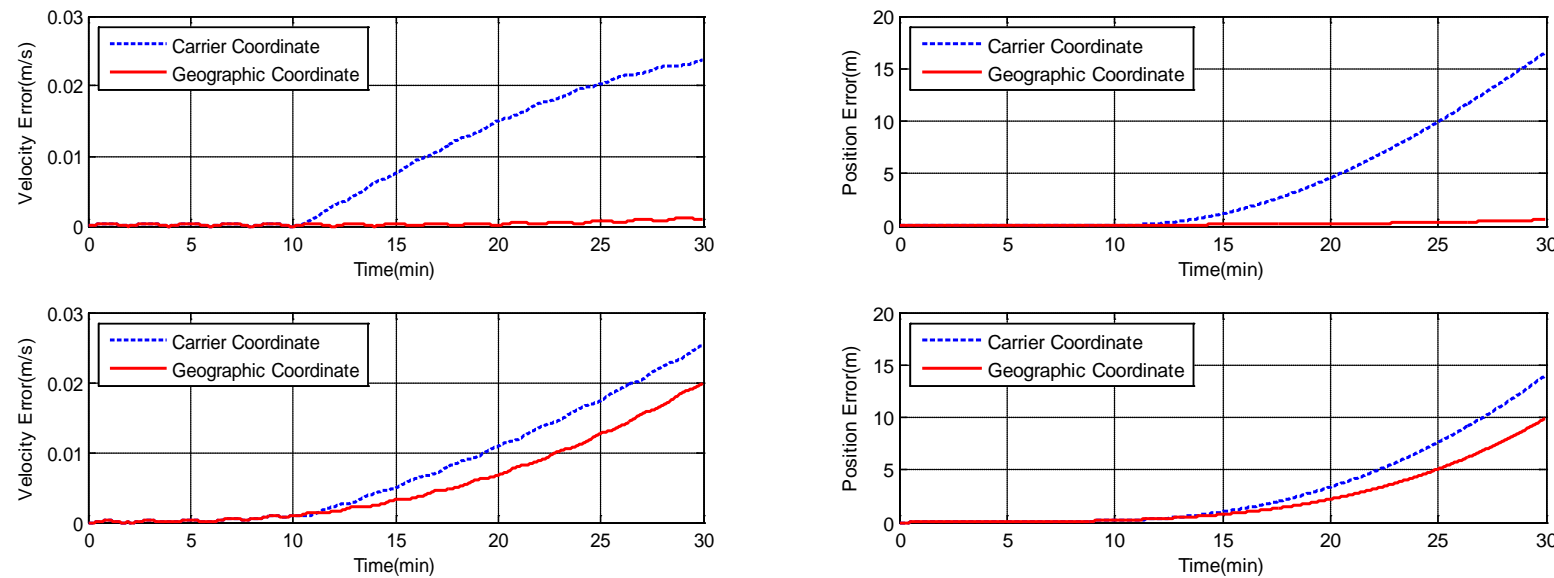

(a)Velocity

(b)Position

Figure 1. Comparison of the navigation results between carrier and geographic coordinate

It is obvious that the rotation scheme of the carrier cause a significant navigation change, when the carrier course is turned. And the rotation scheme of geographic coordinate is not affected by any. Therefore, when the carrier has the attitude change, the effect of rotating around the geographic coordinate is better.

\section{Design of rotation direction}

In order to suppress the influence of the scale factor error, the rotation scheme of single axis RINS is combined with forward and reverse rotation. The most common rotation scheme is turning a 
forward circle and then a reverse circle, so on. The scheme is effective to restrain the equivalent gyro drift caused by the scale factor error, but there are obvious defects in this kind of rotation scheme. In each forward rotation period, the heading attitude error increases from zero to the maximum. And In each reverse rotation period, the heading attitude error increases from maximum to the zero. In the whole process of forward and reverse, attitude is always negative and in a serrated course. When the scale factor error is 10ppm, the serrated heading attitude error is shown in Fig. 2 (a).

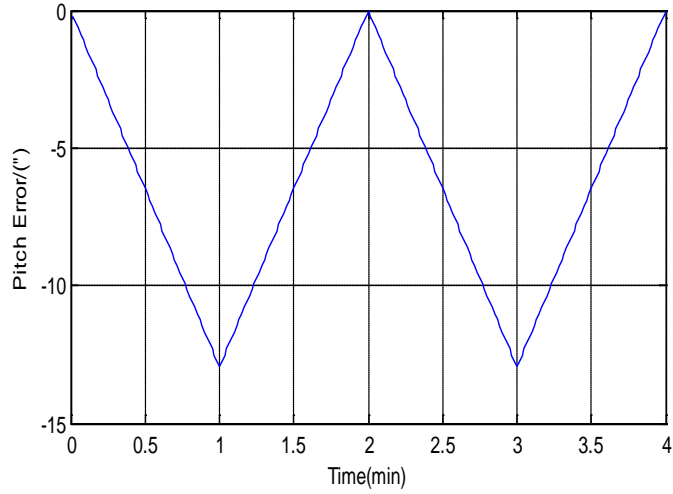

(a)Serrated attitude angle error

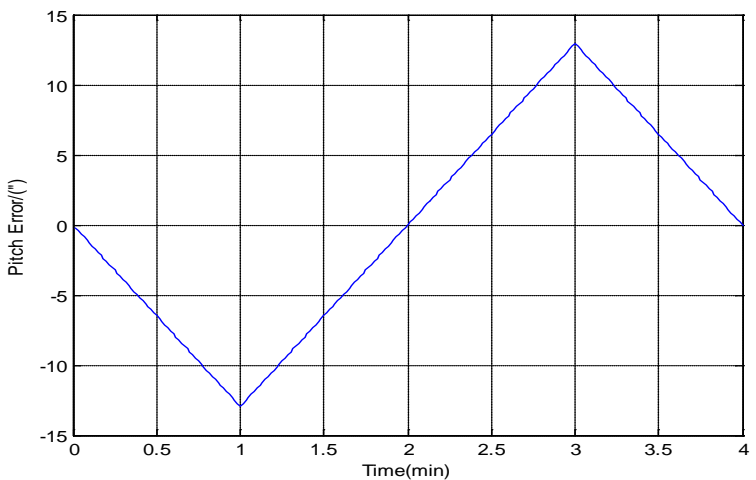

(b)Adjusted angle error

Figure 2. Attitude angle error

Although this error will not lead to divergence of the attitude, it will still obviously affect the navigation accuracy in high precision navigation. Therefore, change the rotation scheme from forward-reverse (named scheme1)to forward-reverse-reverse-forward(named scheme2). In scheme2, the heading attitude error caused by the scale factor error is like Fig. 2(b).

In order to verify the effect of the improved rotation strategy on the scale coefficient error, only the gyro scale factor error is added in the following simulation process.

Simulation condition: Gyro bias $0.01^{\circ} / \mathrm{h}$. IMU $\omega_{1}=6 \%$ s. Simulation time 3 h. The simulation results are as Fig. 3:

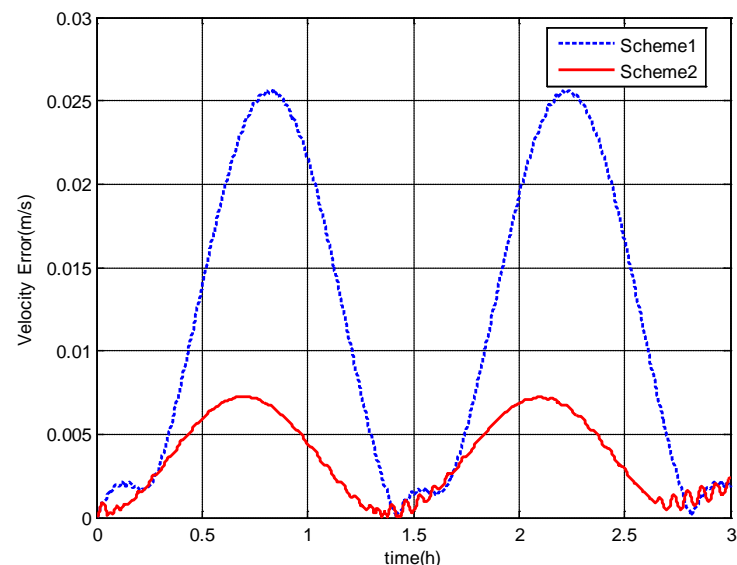

(a)Velocity

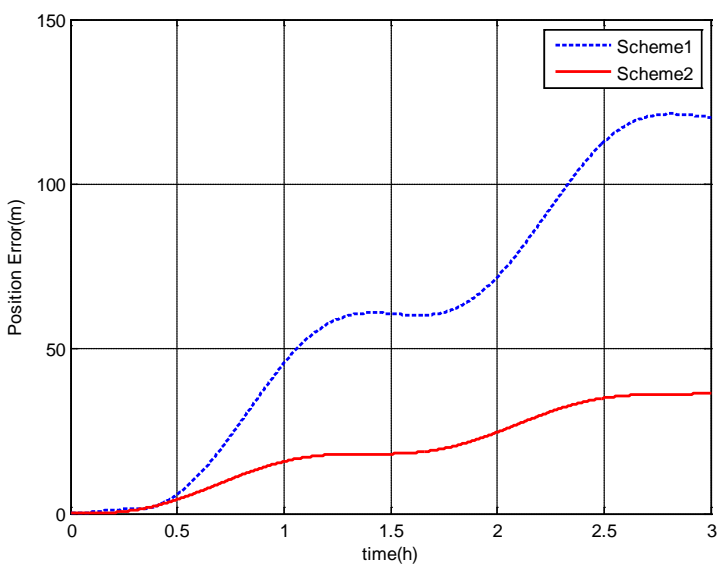

(b)Position

Figure 3. Comparison of scheme 1 and 2 on navigation results

It can be clearly seen that there is a great difference between the navigation accuracy of the two kinds of rotation scheme. In terms of speed and position error, the navigation accuracy of the scheme 2 is increased by 3 times than that of the scheme 1 . In order to show a clear picture, I intercepted a half hour of the simulation results. In terms of the part-graph of the attitude angle, Simulation and theoretical analysis are in good agreement. Improved rotation strategy does improve navigation accuracy. 


\section{Summary}

Starting with modulation heading movement, this paper conducts in-depth analysis of rotating coordinate and scheme aimed at single axis RINS. The simulation results show that the best rotation scheme is to select the geographic coordinate as the rotation of coordinate system and adopt the forward-reverse-reverse- forward rotation scheme. The performance of single axis RINS has been significantly optimized. Navigation accuracy has also been significantly improved.

\section{References}

[1] Ishibashi, S., Tsukioka, S., Yoshida, H., Hyakudome, T., Sawa, T., \& Tahara, J., et al. (2007). Accuracy Improvement of an Inertial Navigation System Brought about by the Rotational Motion. Oceans (Vol.25, pp.1-5).

[2] Zha, F., Jiangning, X. U., \& Qin, F. (2012). Influence of rotation schemes on error of sins. Electronics Optics \& Control.

[3] Liu, X. X., Xiao-Su, X. U., Chen, C., \& Wang, L. H. (2011). Rotation modulation algorithm design for sins based on integration of single-axis rotations. Journal of Chinese Inertial Technology, 19(4), 379-332.

[4] Mao, Y. L., Chen, J. B., Song, C. L., \& Automation, S. O. (2014). Optimal axis selection scheme of sins single-axis rotation modulation. Zhongguo Guanxing Jishu Xuebao/journal of Chinese Inertial Technology, 22(2), 149-156.

[5] Yuan, B. L., \& Rao, G. Y. (2006). On the theory of optical gyro rotating inertial navigation system. Journal of National University of Defense Technology, 28(6), 76-80.

[6] Chang, G. B., Xu, J. N., Li, A., \& Qin, F. J. (2011). Influence of body dynamics on the error of ins scheme with dual-axis continuous autocompensation technique. Journal of Chinese Inertial Technology, 19(2), 175-179. 